\title{
On the Transition of Charlier Polynomials to the Hermite Function
}

\author{
Martin N. P. Nilsson ${ }^{1}$ \\ Received: 27 October 2020 / Accepted: 12 August 2021 / Published online: 23 October 2021 \\ (c) The Author(s) 2021
}

\begin{abstract}
It has been known for over 70 years that there is an asymptotic transition of Charlier polynomials to Hermite polynomials. This transition, which is still presented in its classical form in modern reference works, is valid if and only if a certain parameter is integer. In this light, it is surprising that a much more powerful transition exists from Charlier polynomials to the Hermite function, valid for any real value of the parameter. This greatly strengthens the asymptotic connections between Charlier polynomials and special functions, with applications in queueing theory, where this transition is crucial for solving first-passage problems with moving boundaries. It is shown in this paper that the convergence is locally uniform, and a sharp rate bound is proved. In addition, it is shown that there is a transition of derivatives of Charlier polynomials to the derivative of the Hermite function, again with a sharp rate bound. Finally, it is proved that zeros of Charlier polynomials converge to zeros of the Hermite function.
\end{abstract}

Keywords Orthogonal polynomial $\cdot$ Asymptotic - Uniform convergence $\cdot$ Special function - Sharp rate bound

Mathematics Subject Classification 33C45 - 41A25 · 41A60

\section{Introduction}

A unique feature of Charlier polynomials [1-5] is their affinity with the Poisson distribution. This has many important applications. Charlier polynomials concisely express the behavior of Erlang loss systems, a fundamental concept in queueing theory [6-8]. Here, Charlier polynomials (and the transition to the Hermite function) are instru-

\section{Communicated by Edward B. Saff.}

Martin N. P. Nilsson

martin.nilsson@ri.se

1 RISE Research Institutes of Sweden, Box 1263, 16428 Kista, Sweden 


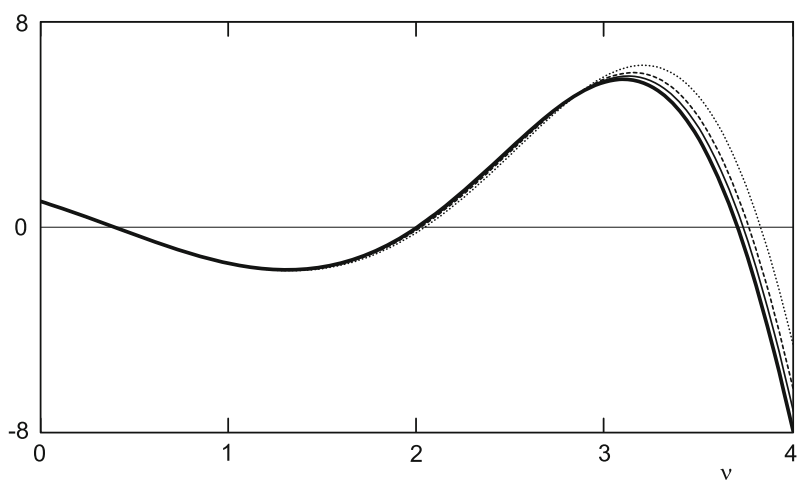

Fig. 1 Transition of Charlier polynomials $(2 a)^{v / 2} C_{\lceil a+\sqrt{a}\rceil}(v, a)$ (thin lines) to the Hermite function $H_{v}(-1 / \sqrt{2})$ (thick line). The different values of the parameter $a$ are 100 (dotted line), 400 (dashed line), and 1600 (solid thin line). Figure adapted from Fig. 2 in [9] under Creative Commons license

mental when computing the probability of the first loss for a time-variable number of servers [9]. Another example is the generalization of stochastic integrals over Poisson distributions to multiple stochastic integrals, which can be effectively computed using Charlier polynomials [10,11], whereas yet another is that of random matrices over Poisson distributions [12], which can be characterized by Charlier polynomial zeros.

High-dimensional or asymptotic problems typically engage Charlier polynomials of high degree and order (index). For instance, the asymptotic behavior in the number of servers of Erlang loss systems is described by Charlier polynomials whose degree and order tend to infinity simultaneously according to the Halfin-Whitt regime [13]. At a first glance, the classical formula ([5, Eq. 9.14.12], [14, p. 532], [4, Eq. 18.21.9], [3, Eq. 2.82.7])

$$
\lim _{a \rightarrow \infty}(2 a)^{n / 2} C_{n}(a+x \sqrt{2 a}, a)=(-1)^{n} H_{n}(x)
$$

appears useful for reducing Charlier polynomials in this limit, but unfortunately, this formula holds only for non-negative integer $n$. In light of the long standing of this formula, it can somewhat surprisingly be shown that

$$
\lim _{a \rightarrow \infty}(2 a)^{v / 2} C_{\lceil a-x \sqrt{2 a}\rceil}(v, a)=H_{\nu}(x)
$$

for any real $x$ and $v$, a much stronger statement (Fig. 1). Here, the ceiling function $\lceil x\rceil$ denotes the smallest integer not smaller than $x$, and $H_{v}(x)$ denotes the Hermite function [15, Ch. 10].

A proof of (1) has been given via Krawtchouk polynomials [3, pp. 36-37]. When $v$ is non-negative real and $a-x \sqrt{2 a}$ is integer, pointwise convergence of (2) (without rate bound) follows implicitly from [16].

In Sect. 2, it is proved rigorously that convergence to the Hermite function holds for any real $v$, and that convergence is uniform for $v$ and $x$ in any bounded interval, i.e., locally uniform. A sharp rate bound is established. The same technique is then 
employed in Sect. 3 to prove that there is a similar transition of the derivative with respect to $v$, and a sharp rate bound is provided here, too. These results are used in Sect. 4 for proving that zeros of Charlier polynomials converge to zeros of the Hermite function.

Below is first a recollection of some well-known definitions and recurrence relations from $[2,4,5,15]$ to make the paper self-contained. This is followed by three sections, each proving an aspect of the transition of Charlier polynomials to Hermite functions.

The notation " $A \triangleq B$ " is used for " $A$ is defined as $B$ ", to make the introduction of new symbols more explicit. The expression "bounded $v \leq-3$ " is shorthand for " $v$ in any bounded interval $\left[v_{0},-3\right]$." Charlier polynomials $C_{n}(x, a)$ will be abbreviated as $c_{n}^{a}(x), c_{n}(x), c_{n}^{a}$, or even $c_{n}$, unless there is a risk for misunderstanding. They can be defined for positive $a$ and non-negative integer $n$ by [2, Eq. 10.25.4], [4, Eq. 18.20.8],

$$
c_{n}^{a}(x) \triangleq \sum_{k=0}^{n}\left(\begin{array}{l}
n \\
k
\end{array}\right)\left(\begin{array}{l}
x \\
k
\end{array}\right) k !(-a)^{-k}
$$

where

$$
\left(\begin{array}{l}
x \\
k
\end{array}\right) \triangleq\left\{\begin{array}{ll}
x(x-1) \cdot \ldots \cdot(x-k+1) / k ! & \text { for } k \geq 1 \\
1 & \text { for } k=0
\end{array} .\right.
$$

These polynomials obey the three-term recurrence relation [2, Eq. 10.25.8], [4, Eq. 18.22.2],

$$
-x c_{n}^{a}(x)=a c_{n+1}^{a}(x)-(n+a) c_{n}^{a}(x)+n c_{n-1}^{a}(x),
$$

and the difference equation [2, Eq. 10.25.9], [4, Eq. 18.22.12],

$$
-n c_{n}^{a}(x)=a c_{n}^{a}(x+1)-(x+a) c_{n}^{a}(x)+x c_{n}^{a}(x-1),
$$

as well as the backward recurrence relation [5, Eq. 9.14.8],

$$
\frac{x}{a} c_{n-1}^{a}(x-1)=c_{n-1}^{a}(x)-c_{n}^{a}(x) .
$$

The Hermite function $H_{v}(x)$ is a solution of the differential equation [15, Eq. 10.2.3]

$$
y^{\prime \prime}=2 x y^{\prime}-2 v y
$$

and satisfies the three-term recurrence [15, Eq. 10.4.7]

$$
H_{v+1}(x)-2 x H_{v}(x)+2 v H_{v-1}(x)=0,
$$

and the derivative rule [15, Eq. 10.4.4]

$$
H_{v}^{\prime}(x)=2 v H_{v-1}(x) .
$$


It can be defined by [15, Eq. 10.2.8]

$$
H_{v}(x) \triangleq 2^{v} \sqrt{\pi}\left[\left(\frac{1}{\Gamma\left(\frac{1-v}{2}\right)}\right) M\left(-\frac{v}{2} ; \frac{1}{2} ; x^{2}\right)-\frac{2 x}{\Gamma\left(-\frac{v}{2}\right)} M\left(\frac{1-v}{2} ; \frac{3}{2} ; x^{2}\right)\right] .
$$

where $M$ is the confluent hypergeometric function of the first kind. When the expression involves a gamma function of a non-positive integer argument, the expression should be interpreted by its limiting value.

\section{Transition of Charlier Polynomials}

Theorem 1 For real $x, v$, and positive $a$,

$$
(2 a)^{v / 2} c_{\lceil a-x \sqrt{2 a}\rceil}^{a}(v)=H_{v}(x)+O\left(\frac{1}{\sqrt{a}}\right),
$$

where $c_{n}^{a}(v)$ are Charlier polynomials and $H_{v}(x)$ is the Hermite function. The error bound $O(1 / \sqrt{a})$ is locally uniform for $v$ and $x$ and is sharp in the sense that there are $v$ and $x$ such that the error is proportional to $1 / \sqrt{a}$ for arbitrarily large a.

Proving asymptotic properties of Charlier polynomials is difficult, since these do not satisfy a second-order linear ordinary differential equation with respect to the independent variable [17]. However, the three-term recurrence relation (4) is a discretization of such a differential equation (7). This can be used to prove the theorem in the following way: It is first proven for the special case $x=0$ and $v \leq-4$ (Lemmas 1-5) and then generalized to arbitrary real $v$ (Lemma 6). After that, the scaled polynomials are shown to approximate a Cauchy polygon converging to the $H_{v}(x)$ solution of the Hermite differential equation initial value problem (Lemma 7).

\subsection{Convergence for $x=0$ and $v \leq-4$}

For notational convenience, define $A \triangleq\lceil a\rceil$ and

$$
y_{v}^{a}(x) \triangleq(2 a)^{v / 2} c_{\lceil a-x \sqrt{2 a}\rceil}^{a}(v) .
$$

The superscript will be left out in $y_{v}^{a}$ and $c_{n}^{a}$ unless there is a risk for misunderstanding. Consider the case $x=0$ and $v \leq-4$. By the definition of Charlier polynomials (3),

$$
c_{n}(v)=\sum_{k=0}^{n}\left(\begin{array}{l}
n \\
k
\end{array}\right) \frac{\Gamma(k-v)}{\Gamma(-v)} a^{-k}
$$


To prove that

$$
\lim _{a \rightarrow \infty} y_{v}(0)=H_{v}(0)=\frac{2^{v} \sqrt{\pi}}{\Gamma\left(\frac{1-v}{2}\right)}
$$

using the definition of $H_{v}(x)$ in $(10), y_{v}(0)$ can be expressed as a sum

$$
y_{\nu}(0)=\frac{2^{v / 2}}{\Gamma(-v)} \sum_{k=0}^{A} T_{k}
$$

of terms

$$
T_{k} \triangleq a^{v / 2} \frac{\Gamma(k-v)}{k !} \frac{A ! a^{-k}}{(A-k) !} .
$$

When $v$ is negative, these are all positive. The series is difficult to sum due to multiple levels of numerical cancellation, but can be estimated by separating the factors. Another difficulty is the changing behavior of $T_{k}$ with increasing $a$. This problem can be remedied by defining a border between "head" and "tail" sections that increases with a properly tuned power of $a$.

Lemma 1 The factor

$$
p(k) \triangleq \frac{A ! a^{-k}}{(A-k) !}=\left(\frac{a}{A}\right)^{-k} \prod_{j=0}^{k-1}\left(1-\frac{j}{A}\right)
$$

for $1 \leq k \leq A$ satisfies

$$
p(k) \leq \exp \left(-\frac{k^{2}}{2 A}\right)\left[1+O\left(\frac{k}{a}\right)\right]
$$

and for $1 \leq k<A / 2$,

$$
p(k) \geq \exp \left(-\frac{k^{2}}{2 A}\right)\left[1+O\left(\frac{k}{a}+\frac{k^{3}}{a^{2}}\right)\right] .
$$

Proof Define the "nuisance factor" due to truncation by the ceiling function by

$$
\beta \triangleq\left(\frac{a}{A}\right)^{k}=\left(\frac{a}{a+(\lceil a\rceil-a)}\right)^{k}=\left(\frac{a}{a+\theta}\right)^{k}=1+O\left(\frac{k}{a}\right)
$$

where $0 \leq \theta<1$. For $1 \leq k \leq A$, taking the logarithm of $\beta p(k)$ and Taylor expanding, 


$$
\begin{aligned}
\ln [\beta p(k)] & =\sum_{j=0}^{k-1} \ln \left(1-\frac{j}{A}\right) \\
& =\sum_{j=0}^{k-1}\left(-\frac{j}{A}-\frac{j^{2}}{2 A^{2}}-\frac{j^{3}}{3 A^{3}}-\frac{j^{4}}{4 A^{4}}-\cdots\right) \\
& =-\frac{k(k-1)}{2 A}-\sum_{j=0}^{k-1}\left(\frac{j^{2}}{2 A^{2}}+\frac{j^{3}}{3 A^{3}}+\frac{j^{4}}{4 A^{4}}+\cdots\right) \\
& \triangleq-\frac{k(k-1)}{2 A}-R_{p},
\end{aligned}
$$

where $R_{p} \geq 0$. By re-exponentiation,

$\beta p(k) \leq \exp \left(-\frac{k(k-1)}{2 A}\right)=\exp \left(-\frac{k^{2}}{2 A}+\frac{k}{2 A}\right)=\exp \left(-\frac{k^{2}}{2 A}\right)\left[1+O\left(\frac{k}{a}\right)\right]$

so

$$
p(k) \leq \exp \left(-\frac{k^{2}}{2 A}\right)\left[1+O\left(\frac{k}{a}\right)\right] .
$$

On the other hand, for $1 \leq k \leq A / 2$, by comparison with a geometric series,

$$
\begin{aligned}
R_{p} & =\sum_{j=0}^{k-1}\left(\frac{j^{2}}{2 A^{2}}+\frac{j^{3}}{3 A^{3}}+\frac{j^{4}}{4 A^{4}} \cdots\right) \\
& \leq \sum_{j=0}^{k-1}\left(\frac{j^{2}}{2 A^{2}}+\frac{j^{3}}{2 A^{3}}+\frac{j^{4}}{2 A^{4}} \cdots\right) \\
& =\sum_{j=0}^{k-1}\left(\frac{j^{2}}{2 A^{2}} \frac{1}{1-j / A}\right) \leq \sum_{j=0}^{k-1} \frac{j^{2}}{A^{2}} \leq \frac{k^{3}}{A^{2}},
\end{aligned}
$$

so by (14),

$$
\beta p(k) \geq \exp \left(-\frac{k(k-1)}{2 A}-\frac{k^{3}}{A^{2}}\right) \geq \exp \left(-\frac{k^{2}}{2 A}-\frac{k^{3}}{A^{2}}\right),
$$

By (13)

$$
\begin{aligned}
p(k) & \geq \exp \left(-\frac{k^{2}}{2 A}-\frac{k^{3}}{A^{2}}\right)\left[1+O\left(\frac{k}{a}\right)\right] \\
& =\exp \left(-\frac{k^{2}}{2 A}\right) \exp \left(-\frac{k^{3}}{A^{2}}\right)\left[1+O\left(\frac{k}{a}\right)\right]
\end{aligned}
$$




$$
=\exp \left(-\frac{k^{2}}{2 A}\right)\left[1+O\left(\frac{k}{a}+\frac{k^{3}}{a^{2}}\right)\right]
$$

The following lemma is similar to Gautschi's inequality [18], but whereas the latter inequality is restricted to $-1 \leq v \leq 0$, the lemma here needs to hold for arbitrary negative $v$.

\section{Lemma 2 The factor}

$$
q(k) \triangleq \frac{\Gamma(k-v)}{k !}
$$

for $1 \leq k \leq A$ and $v \leq 0$ satisfies

$$
q(k)=k^{-v-1}\left[1+O\left(\frac{1}{k}\right)\right] .
$$

Proof By Stirling's approximation for $k>0$ [19, §6.1.37-38], [4, Eq. 5.11.3]

$$
\Gamma(k)=\sqrt{\frac{2 \pi}{k}}\left(\frac{k}{e}\right)^{k}\left[1+O\left(\frac{1}{k}\right)\right],
$$

and the relation

$$
\left(1-\frac{v}{k}\right)^{k}=\exp \left[k \ln \left(1-\frac{v}{k}\right)\right]=\exp \left[-v+O\left(\frac{1}{k}\right)\right]=e^{-v}\left[1+O\left(\frac{1}{k}\right)\right]
$$

gives

$$
\begin{aligned}
\frac{\Gamma(k-v)}{\Gamma(k)} & =\sqrt{\frac{k}{k-v}}\left(\frac{k-v}{e}\right)^{k-v}\left(\frac{e}{k}\right)^{k}\left[1+O\left(\frac{1}{k}\right)\right] \\
& =\left(1-\frac{v}{k}\right)^{-\frac{1}{2}-v}\left(1-\frac{v}{k}\right)^{k} e^{v} k^{-v}\left[1+O\left(\frac{1}{k}\right)\right] \\
& =\left[1+O\left(\frac{1}{k}\right)\right] e^{-v} e^{v} k^{-v}\left[1+O\left(\frac{1}{k}\right)\right] \\
& =k^{-v}\left[1+O\left(\frac{1}{k}\right)\right],
\end{aligned}
$$

so from the definition of $q(k)$,

$$
q(k)=\frac{\Gamma(k-v)}{k !}=\frac{\Gamma(k-v)}{k \Gamma(k)}=k^{-v-1}\left[1+O\left(\frac{1}{k}\right)\right] .
$$




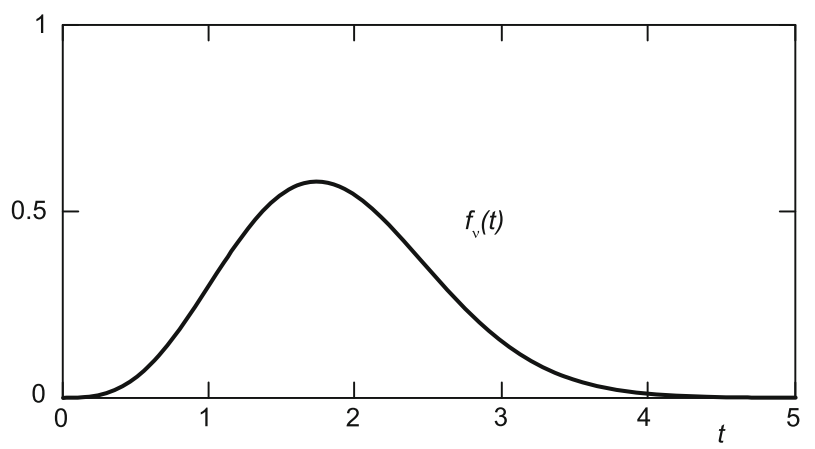

Fig. 2 The function $f_{v}(t)$ for $v=-4$

Now, it is time to take on the sum (12), split in a head and tail part at index $M \triangleq\left\lceil A^{3 / 4}\right\rceil$,

$$
\sum_{k=0}^{A} T_{k}=\sum_{k=0}^{M-1} T_{k}+\sum_{k=M}^{A} T_{k} \triangleq R_{\text {head }}+R_{\text {tail }}
$$

Define $\Delta t \triangleq 1 / \sqrt{A}$ and the function

$$
f_{v}(t) \triangleq t^{-v-1} \exp \left(-\frac{t^{2}}{2}\right)
$$

Clearly, the functions $f_{v}(t)$ (Fig. 2) and

$$
f_{v}^{\prime \prime}(t)=\left[t^{4}+(1+2 v) t^{2}+\left(v^{2}+3 v+2\right)\right] t^{-v-3} e^{-t^{2} / 2}
$$

are continuous and bounded for bounded $v \leq-3$ and $t \geq 0$.

Lemma 3 The following relations hold for $v \leq-3$ :

$$
\sum_{k=M}^{A} f_{v}(k \Delta t) \Delta t=O\left(\frac{1}{\sqrt{a}}\right)
$$

and

$$
\sum_{k=0}^{A} f_{v}(k \Delta t) \Delta t=2^{-v / 2-1} \Gamma\left(-\frac{v}{2}\right)+O\left(\frac{1}{\sqrt{a}}\right) .
$$


Proof According to the well-known trapezoidal rule, since $f_{v}(t)$ and $f_{v}^{\prime \prime}(t)$ are bounded for $v \leq-3$ and $t \geq 0$, and for some $\tau \in[M \Delta t, A \Delta t]$,

$$
\begin{aligned}
\int_{M \Delta t}^{A \Delta t} f_{v}(t) \mathrm{d} t= & \left(\frac{f_{v}(A \Delta t)}{2}+\frac{f_{v}(M \Delta t)}{2}\right) \Delta t \\
& +\sum_{k=M+1}^{A-1} f_{v}(k \Delta t) \Delta t-\frac{A \Delta t-M \Delta t}{12} \Delta t^{2} f_{v}^{\prime \prime}(\tau) \\
= & \frac{f_{v}(A \Delta t)+f_{v}(M \Delta t)}{2} \Delta t+\sum_{k=M+1}^{A-1} f_{v}(k \Delta t) \Delta t+O\left(A \Delta t^{3}\right),
\end{aligned}
$$

so that

$$
\sum_{k=M}^{A} f_{v}(k \Delta t) \Delta t=\int_{M \Delta t}^{A \Delta t} f_{v}(t) \mathrm{d} t+O\left(\frac{1}{\sqrt{a}}\right)
$$

By substituting $t^{2} / 2=u$ in the integral of $f_{v}$, the upper incomplete gamma function $[19, \S 6.5 .3]$, [4, Eq. 8.2.2] is obtained,

$$
\begin{aligned}
\int f_{v}(t) \mathrm{d} t & =\int t^{-v-1} e^{-\frac{t^{2}}{2}} \mathrm{~d} t \\
& =\int(\sqrt{2 u})^{-v-2} e^{-u} \mathrm{~d} u \\
& =2^{-v / 2-1} \int u^{-v / 2-1} e^{-u} \mathrm{~d} u \\
& =-2^{-v / 2-1} \Gamma\left(-\frac{v}{2}, u\right)+C .
\end{aligned}
$$

Asymptotically [19, §6.5.32], [4, Eq. 8.11.2-3],

$$
\Gamma(s, z)=z^{s-1} e^{-z}\left[1+O\left(\frac{1}{z}\right)\right],
$$

implying that when $z$ increases, $\Gamma(s, z)$ approaches zero faster than any negative power of $z$, including $1 / \sqrt{a}$, i.e.,

$$
\Gamma\left(-\frac{v}{2}, \frac{(M \Delta t)^{2}}{2}\right)=O\left(\frac{1}{\sqrt{a}}\right) .
$$


This proves the first relation. For the second relation, by (18),

$$
\begin{aligned}
\int_{0}^{A \Delta t} f_{v}(t) \mathrm{d} t & =2^{-v / 2-1} \Gamma\left(-\frac{v}{2}\right)-2^{-v / 2-1} \Gamma\left(-\frac{v}{2}, \frac{(A \Delta t)^{2}}{2}\right) \\
& =2^{-v / 2-1} \Gamma\left(-\frac{v}{2}\right)+O\left(\frac{1}{\sqrt{a}}\right) .
\end{aligned}
$$

Lemma 4 For bounded $v \leq-3, R_{\text {tail }}=O(1 / \sqrt{a})$.

Proof By Lemmas 1 and 2,

$$
\begin{aligned}
0<R_{\text {tail }} & =a^{v / 2} \sum_{k=M}^{A} q(k) p(k) \\
& \leq a^{v / 2} \sum_{k=M}^{A} k^{-v-1}\left[1+O\left(\frac{1}{k}\right)\right] e^{-k^{2} / 2 A}\left[1+O\left(\frac{k}{a}\right)\right] \\
& =a^{v / 2} \sum_{k=M}^{A} k^{-v-1} e^{-k^{2} / 2 A} O(1) .
\end{aligned}
$$

Substituting $k=k \Delta t \sqrt{A}$,

$$
\begin{aligned}
R_{\text {tail }} & =a^{\nu / 2} \sum_{k=M}^{A}(k \Delta t \sqrt{A})^{-v-1} e^{-(k \Delta t)^{2} / 2} \Delta t \sqrt{A} \cdot O(1) \\
& =\left(\frac{a}{A}\right)^{\frac{\nu}{2}} \sum_{k=M}^{A} f_{v}(k \Delta t) \Delta t \cdot O(1) \\
& =O\left(\frac{1}{\sqrt{a}}\right)
\end{aligned}
$$

by Lemma 3.

The term $R_{\text {head }}$ in (15) can be computed in a similar way.

Lemma 5 For bounded $v \leq-4$,

$$
R_{\text {head }}=2^{-v / 2-1} \Gamma\left(-\frac{v}{2}\right)+O\left(\frac{1}{\sqrt{a}}\right) .
$$

Proof This time $k<M$, and by Lemmas 1 and 2 ,

$$
R_{\text {head }}=a^{\nu / 2} \sum_{k=0}^{M-1} k^{-v-1}\left[1+O\left(\frac{1}{k}\right)\right] e^{-\frac{k^{2}}{2 A}}\left[1+O\left(\frac{k}{a}+\frac{k^{3}}{a^{2}}\right)\right]
$$




$$
\begin{aligned}
& =\left(\frac{a}{A}\right)^{v / 2} \sum_{k=0}^{M-1} f_{v}(k \Delta t) \Delta t\left[1+O\left(\frac{1}{k}+\frac{k}{a}+\frac{k^{3}}{a^{2}}\right)\right] \\
& =\sum_{k=0}^{M-1} f_{v}(k \Delta t) \Delta t\left[1+O\left(\frac{1}{a}+\frac{\Delta t}{k \Delta t}+\frac{k \Delta t}{\sqrt{a}}+\frac{(k \Delta t)^{3}}{\sqrt{a}}\right)\right] \\
& =\sum_{k=0}^{M-1} f_{v}(k \Delta t) \Delta t+\sum_{k=0}^{M-1} f_{v}(k \Delta t) \Delta t \cdot O\left(\frac{\Delta t}{k \Delta t}+\frac{k \Delta t}{\sqrt{a}}+\frac{(k \Delta t)^{3}}{\sqrt{a}}\right) \\
& \triangleq S+\Delta S .
\end{aligned}
$$

Using the identity $f_{v}(t) t^{n}=f_{v-n}(t)$, the error term $\Delta S$ is

$$
\begin{aligned}
\Delta S= & \sum_{k=0}^{M-1} f_{v+1}(k \Delta t) \Delta t \cdot O(\Delta t) \\
& +\sum_{k=0}^{M-1} f_{v-1}(k \Delta t) \Delta t \cdot O(1 / \sqrt{a}) \\
& +\sum_{k=0}^{M-1} f_{v-3}(k \Delta t) \Delta t \cdot O(1 / \sqrt{a}) .
\end{aligned}
$$

Since

$$
0 \leq \sum_{k=0}^{M-1} f_{v}(k \Delta t) \Delta t \leq \sum_{k=0}^{A} f_{v}(k \Delta t) \Delta t
$$

which by Lemma 3 is bounded for $v \leq-3$,

$$
\Delta S=O\left(\frac{1}{\sqrt{a}}\right)
$$

for $v \leq-4$. For the sum $S$ in (20), again using Lemma 3,

$$
\begin{aligned}
S & =\sum_{k=0}^{M-1} f_{v}(k \Delta t) \Delta t \\
& =\sum_{k=0}^{A} f_{v}(k \Delta t) \Delta t-\sum_{k=M}^{A} f_{v}(k \Delta t) \Delta t \\
& =2^{-v / 2-1} \Gamma\left(-\frac{v}{2}\right)+O\left(\frac{1}{\sqrt{a}}\right) .
\end{aligned}
$$


By (12), and combining Lemmas 5 and 4,

$$
y_{v}^{a}(0)=\frac{2^{v / 2}}{\Gamma(-v)}\left(R_{\text {head }}+R_{\text {tail }}\right)=\frac{\Gamma\left(-\frac{v}{2}\right)}{2 \Gamma(-v)}+O\left(\frac{1}{\sqrt{a}}\right) .
$$

By the gamma function duplication rule [15, Eq. 1.2.3], [4, Eq. 5.5.5],

$$
\frac{\Gamma(z)}{\Gamma(2 z)}=\frac{2^{1-2 z} \sqrt{\pi}}{\Gamma\left(z+\frac{1}{2}\right)}
$$

substituting $z=-v / 2$,

$$
y_{v}^{a}(0)=\frac{2^{v} \sqrt{\pi}}{\Gamma\left(\frac{1-v}{2}\right)}+O\left(\frac{1}{\sqrt{a}}\right)=H_{v}(0)+O\left(\frac{1}{\sqrt{a}}\right) .
$$

\subsection{Convergence for $x=0$ and Arbitrary $v$}

Lemma 6 For $v$ in any bounded interval,

$$
y_{v}(0)=H_{v}(0)+O\left(\frac{1}{\sqrt{a}}\right)
$$

and for $\Delta x=1 / \sqrt{2 a}$,

$$
\frac{y_{v}(0)-y_{v}(-\Delta x)}{\Delta x}=H_{v}^{\prime}(0)+O\left(\frac{1}{\sqrt{a}}\right) .
$$

Proof Given that $y_{v}(0)=H_{v}(0)+O(1 / \sqrt{a})$ for bounded $v<v_{0}$, then for $v_{0} \leq v<$ $v_{0}+1$ the difference equation (5) can be rewritten into

$$
c_{n}(v+1)=\frac{v+a-n}{a} c_{n}(v)-\frac{v}{a} c_{n}(v-1),
$$

so that for $n=A=\lceil a\rceil$ and by (21),

$$
\begin{aligned}
(2 a)^{(v+1) / 2} c_{A}(v+1) & =(2 a)^{(v+1) / 2}\left[\frac{v+a-A}{a} c_{A}(v)-\frac{v}{a} c_{A}(v-1)\right] \\
& =\sqrt{2 a} \frac{v+a-A}{a} y_{v}(0)-\frac{v}{a} 2 a y_{v-1}(0) \\
& =O\left(\frac{1}{\sqrt{a}}\right)-2 v y_{v-1}(0) \\
& =-2 v H_{v}(0)+O\left(\frac{1}{\sqrt{a}}\right) \\
& =H_{v+1}(0)+O\left(\frac{1}{\sqrt{a}}\right) .
\end{aligned}
$$


By induction, $y_{v}(0)=H_{v}(0)+O(1 / \sqrt{a})$ locally for $v$. Additionally, by the backward recurrence relation (6) and the derivative rule for the Hermite function (9),

$$
\begin{aligned}
\frac{y_{v}(0)-y_{v}(-\Delta x)}{\Delta x} & =\frac{(2 a)^{v / 2} c_{A}(v)-(2 a)^{v / 2} c_{A+1}(v)}{1 / \sqrt{2 a}} \\
& =\frac{v}{a}(2 a)^{v / 2+1 / 2} c_{A}(v-1) \\
& =2 v y_{v-1}(0) \\
& =2 v H_{v-1}(0)+O\left(\frac{1}{\sqrt{a}}\right) \\
& =H_{v}^{\prime}(0)+O\left(\frac{1}{\sqrt{a}}\right) .
\end{aligned}
$$

\subsection{Convergence for Arbitrary $x$ and Arbitrary $v$}

To prove that $y_{\nu}(x)$ in (11) converges to the solution of the Hermite differential equation (7) having initial conditions $y(0)=H_{v}(0)$ and $y^{\prime}(0)=H_{v}^{\prime}(0)$, it can be rewritten in normal form as

$$
\boldsymbol{y}^{\prime}=\boldsymbol{A}(x) \boldsymbol{y}
$$

where $\boldsymbol{y}(x) \triangleq\left(y(x), y^{\prime}(x)\right)^{T}$ and

$$
\boldsymbol{A}(x) \triangleq\left(\begin{array}{cc}
0 & 1 \\
-2 v & 2 x
\end{array}\right) .
$$

Let $r \triangleq \sqrt{2 a}, \Delta x \triangleq 1 / r$, and $x_{k} \triangleq k \Delta x$. Define a Cauchy polygon $\boldsymbol{u}(x)$ for the differential equation (25) by linear interpolation between points $\left(x_{k}, \boldsymbol{u}_{k}\right)$, where $\boldsymbol{u}_{0}=$ $\boldsymbol{y}(0)$ and

$$
\boldsymbol{u}_{k+1} \triangleq \boldsymbol{u}_{k}+\Delta x \boldsymbol{A}\left(x_{k}\right) \boldsymbol{u}_{k}
$$

Lemma 7 For $x$ and $v$ in bounded intervals $[0, \xi]$ and $[-\psi, \psi]$, respectively, the Cauchy polygon $\boldsymbol{u}(x)$ converges uniformly to the Hermite function solution with an error bound

$$
|\boldsymbol{u}(x)-\boldsymbol{y}(x)| \leq O\left(\frac{1}{\sqrt{a}}\right) .
$$

Proof The Euclidean norm $\|\boldsymbol{A}(x)\|$ of $\boldsymbol{A}$ in (25) equals the largest singular value of the matrix, so

$$
\|\boldsymbol{A}(x)\|=\sigma_{\max }(\boldsymbol{A}(x)) \leq \sqrt{\operatorname{tr}\left(\boldsymbol{A}(x)^{T} \boldsymbol{A}(x)\right)}=\sqrt{1+4 v^{2}+4 x^{2}}
$$


Given arbitrary $\xi, \psi>0$ and $L \triangleq \sqrt{1+4 \psi^{2}+4 \xi^{2}}$, for $x$ in $[0, \xi]$ and $\nu$ in $[-\psi, \psi]$, by the definition of the Euclidean norm,

$$
\frac{|\boldsymbol{A}(x)(\boldsymbol{y}-\boldsymbol{z})|}{|\boldsymbol{y}-\boldsymbol{z}|} \leq\|\boldsymbol{A}(x)\| \leq L,
$$

so $L$ is additionally a Lipschitz constant for (25) when $x \in[0, \xi]$. A definition and two theorems proved in [20, Sect. 7.3] are now handy:

Definition 1 A vector function $\boldsymbol{u}(x)$ is an approximate solution with deviation at most $\epsilon$ in the interval $a \leq x \leq \xi+a$ of the vector differential equation

$$
\mathrm{d} \boldsymbol{y} / \mathrm{d} x=\boldsymbol{Y}(\boldsymbol{y}, x), \quad a \leq x \leq a+\xi,
$$

when $\boldsymbol{u}(x)$ is continuous and satisfies the differential inequality

$$
\left|\boldsymbol{u}^{\prime}(x)-\boldsymbol{Y}(\boldsymbol{u}(x), x)\right| \leq \epsilon
$$

for all except a finite number of points $x$ of the interval $[a, a+\xi]$.

Theorem 2 (Birkhoff and Rota, Th. 7.1) Let the continuously differentiable function $\boldsymbol{Y}$ satisfy $|\boldsymbol{Y}| \leq M,|\partial \boldsymbol{Y} / \partial x| \leq C$, and $L$ be a Lipschitz constant in the cylinder $D:|\boldsymbol{y}-\boldsymbol{c}| \leq K, a \leq x \leq a+\xi$. Then, any Cauchy polygon in $D$ with partition $\pi$ is an approximate solution of $\boldsymbol{y}^{\prime}(x)=\boldsymbol{Y}(\boldsymbol{y}, x)$ with deviation at most $(C+L M)|\pi|$.

Theorem 3 (Birkhoff and Rota, Th. 7.3) Let $\boldsymbol{y}(x)$ be an exact solution and $\boldsymbol{u}(x)$ be an approximate solution, with deviation $\epsilon$, of the differential equation $\boldsymbol{y}^{\prime}(x)=\boldsymbol{Y}(\boldsymbol{y}, x)$. Let $\boldsymbol{Y}$ satisfy a Lipschitz condition with Lipschitz constant L. Then, for $x \geq a$,

$$
|\boldsymbol{y}(x)-\boldsymbol{u}(x)| \leq|\boldsymbol{y}(a)-\boldsymbol{u}(a)| e^{L(x-a)}+(\epsilon / L)\left(e^{L(x-a)}-1\right) .
$$

Bounds for $|\boldsymbol{A}(x) \boldsymbol{u}(x)|$ and $|\partial(\boldsymbol{A}(x) \boldsymbol{u}(x)) / \partial x|$ in $[0, \xi]$ can be chosen

$$
\left|\boldsymbol{A}\left(x_{k}\right) \boldsymbol{u}_{k}\right| \leq L\left|\boldsymbol{u}_{k}\right| \leq L\left|\boldsymbol{u}_{0}\right| \prod_{j=0}^{k-1}\left\|I+\Delta x \boldsymbol{A}\left(x_{j}\right)\right\| \leq L\left|\boldsymbol{u}_{0}\right|(1+L \Delta x)^{k} \leq L\left|\boldsymbol{u}_{0}\right| e^{L \xi} \triangleq M
$$

and

$$
\left|\frac{\partial(\boldsymbol{A}(x) \boldsymbol{u}(x))}{\partial x}\right|_{x=x_{k}}=\left|\left(\begin{array}{ll}
0 & 0 \\
0 & 2
\end{array}\right) \boldsymbol{u}_{k}\right| \leq 2\left|\boldsymbol{u}_{k}\right| \leq 2\left|\boldsymbol{u}_{0}\right| e^{L \xi} \triangleq C .
$$

By Theorem 2, Theorem 3, and Lemma 6, for $x \in[0, \xi]$ and $v \in[-\psi, \psi]$,

$$
|\boldsymbol{u}(x)-\boldsymbol{y}(x)| \leq|\boldsymbol{u}(0)-\boldsymbol{y}(0)| e^{L x}+\Delta x\left(\frac{C}{L}+M\right)\left(e^{L x}-1\right)
$$




$$
\begin{aligned}
& =O\left(\frac{1}{\sqrt{a}}\right) e^{L \xi}+\Delta x\left(\frac{2}{L}+L\right)\left|\boldsymbol{u}_{0}\right| e^{L \xi}\left(e^{L \xi}-1\right) \\
& =O\left(\frac{1}{\sqrt{a}}\right)
\end{aligned}
$$

which is independent of $x$ and $v$, so the Cauchy polygon (26) converges uniformly to the Hermite function when $a \rightarrow \infty$.

Define $z_{0} \triangleq \boldsymbol{u}_{0}$ and

$$
\begin{aligned}
z_{k+1} & \triangleq\left(\begin{array}{c}
y_{v}\left(x_{k+1}\right) \\
\frac{y_{v}\left(x_{k+1}\right)-y_{v}\left(x_{k}\right)}{\Delta x}
\end{array}\right) \\
& =z_{k}+\Delta x\left(\begin{array}{c}
\frac{y_{v}\left(x_{k+1}\right)-y_{v}\left(x_{k}\right)}{\Delta x} \\
\frac{y_{v}\left(x_{k+1}\right)-2 y_{v}\left(x_{k}\right)+y_{v}\left(x_{k-1}\right)}{\Delta x^{2}}
\end{array}\right) .
\end{aligned}
$$

Let $m \triangleq\left\lceil a-x_{k} r\right\rceil=a-x_{k} r+(\lceil a\rceil-a)=a-x_{k} r+\theta$, where $0 \leq \theta<1$. For simplicity of notation, the argument of $c_{m}$ is dropped when it is $v$. Consequently,

$$
\boldsymbol{z}_{k}=\left(\begin{array}{c}
r^{\nu} c_{m} \\
r^{\nu+1}\left(c_{m-1}-c_{m}\right)
\end{array}\right)
$$

and

$$
z_{k+1}=z_{k}+\Delta x\left(\begin{array}{c}
r^{\nu+1}\left(c_{m-1}-c_{m}\right) \\
r^{\nu+2}\left(c_{m-1}-2 c_{m}+c_{m+1}\right)
\end{array}\right) .
$$

Multiplying the three-term recurrence relation (4) by two, and substituting $x=v$ and $m=n$ gives the identity

$$
-2 v c_{m}=2 a c_{m+1}-(2 m+2 a) c_{m}+2 m c_{m-1} .
$$

Rearranging, and using the facts that $2 a=r^{2}$ and $m=a-x_{k} r+\theta$,

$$
r^{2} c_{m-1}-2 r^{2} c_{m}+r^{2} c_{m+1}=2 x_{k} r\left(c_{m-1}-c_{m}\right)-2 \nu c_{m}-2 \theta\left(c_{m-1}-c_{m}\right),
$$

by which

$$
\begin{aligned}
z_{k+1} & =z_{k}+\Delta x\left(\begin{array}{c}
r^{\nu+1}\left(c_{m-1}-c_{m}\right) \\
2\left(x_{k}-\theta / r\right) r^{\nu+1}\left(c_{m-1}-c_{m}\right)-2 v r^{\nu} c_{m}
\end{array}\right) \\
& =z_{k}+\Delta x\left(\begin{array}{cc}
0 & 1 \\
-2 v & 2 x_{k}-2 \theta / r
\end{array}\right) z_{k} \\
& =z_{k}+\Delta x \boldsymbol{A}\left(x_{k}\right) z_{k}+\Delta x\left(\begin{array}{cc}
0 & 0 \\
0 & -2 \theta / r
\end{array}\right) z_{k} .
\end{aligned}
$$


This is nearly the same expression as for the Cauchy polygon (26), with only the $\theta$-term differing. Understanding the product sign below to multiply matrices in the proper order, and $\boldsymbol{I}$ to denote the identity matrix,

$$
\frac{\left|z_{k+1}-\boldsymbol{u}_{k+1}\right|}{\left|\boldsymbol{u}_{0}\right|} \leq\left\|\prod_{j=0}^{k}\left[\boldsymbol{I}+\Delta x \boldsymbol{A}\left(x_{j}\right)+\Delta x\left(\begin{array}{cc}
0 & 0 \\
0 & -2 \theta / r
\end{array}\right)\right]-\prod_{j=0}^{k}\left[\boldsymbol{I}+\Delta x \boldsymbol{A}\left(x_{j}\right)\right]\right\| .
$$

Bounding the factor $\left\|\boldsymbol{I}+\Delta x \boldsymbol{A}\left(x_{j}\right)\right\| \leq \exp (L \xi)$ in the same way as in (27),

$$
\begin{aligned}
\frac{\left|\boldsymbol{z}_{k+1}-\boldsymbol{u}_{k+1}\right|}{\left|\boldsymbol{u}_{0}\right|} & \leq \sum_{j=1}^{k}\left(\begin{array}{l}
k \\
j
\end{array}\right) \Delta x^{j}\left\|\left(\begin{array}{cc}
0 & 0 \\
0 & -2 \theta / r
\end{array}\right)\right\|^{j} e^{L \xi} \\
& =\sum_{j=1}^{k}\left(\begin{array}{l}
k \\
j
\end{array}\right)\left(\frac{2 \theta \Delta x}{r}\right)^{j} e^{L \xi}=O\left(\frac{\xi}{r}\right) e^{L \xi}=O\left(\frac{1}{\sqrt{a}}\right),
\end{aligned}
$$

demonstrates that $z$ converges uniformly to $\boldsymbol{u}$ for $x \in[0, \xi]$ and $v \in[-\psi, \psi]$. The proof for the descending direction from $x=0$ is omitted, since it is exactly analogous. By (28) and Lemma 7,

$$
\left|z_{k}-\boldsymbol{y}\left(x_{k}\right)\right| \leq\left|z_{k}-\boldsymbol{u}_{k}\right|+\left|\boldsymbol{u}\left(x_{k}\right)-\boldsymbol{y}\left(x_{k}\right)\right|=O\left(\frac{1}{\sqrt{a}}\right)
$$

so for $x_{k} \leq x<x_{k+1}$,

$$
\begin{aligned}
\left|y_{v}^{a}(x)-H_{v}(x)\right| & \leq\left|y_{v}^{a}\left(x_{k}\right)-H_{v}\left(x_{k}\right)\right|+\left|y_{v}^{a}\left(x_{k}\right)-y_{v}^{a}\left(x_{k+1}\right)\right| \\
& \leq\left|z_{k}-\boldsymbol{y}\left(x_{k}\right)\right|+\left|z_{k}-z_{k+1}\right| \\
& =O\left(\frac{1}{\sqrt{a}}\right),
\end{aligned}
$$

where the right hand side is independent of $x$ and $v$ for these parameters in any bounded interval.

To demonstrate the sharpness of the bound, choose $v=2$, any real $x$, and arbitrarily large $a$ such that $n=a-x \sqrt{2 a}$ is integer. Since $c_{2}^{a}(n)=1-(1+2 a) n / a^{2}+n^{2} / a^{2}$ and $H_{2}(x)=4 x^{2}-2$,

$$
y_{2}^{a}(x)-H_{2}(x)=\frac{2 x \sqrt{2}}{\sqrt{a}} .
$$

This completes the proof of Theorem 1 . 


\section{Transition of the Derivative}

Theorem 4 For real $x, v$, and positive $a$,

$$
\frac{\partial}{\partial v}\left\{(2 a)^{v / 2} c_{\lceil a-x \sqrt{2 a}\rceil}^{a}(v)\right\}=\frac{\partial}{\partial v} H_{v}(x)+O\left(\frac{1}{\sqrt{a}}\right),
$$

where $c_{n}^{a}(v)$ are Charlier polynomials and $H_{v}(x)$ is the Hermite function. The error bound $O(1 / \sqrt{a})$ is sharp and locally uniform for $v$ and $x$.

The proof of this theorem uses the same technique as the proof of Theorem 1, so the procedure is abbreviated. First, the theorem is proved for the special case $x=0$ and $v \leq 5$, then generalized to arbitrary $v$, and finally shown to converge to the solution of a differential equation uniquely solved by the derivative of the Hermite function.

\subsection{Convergence for $x=0$ and $v \leq-5$}

Differentiating (12) with respect to $v$,

$$
\frac{\partial y_{v}(0)}{\partial v}=\frac{\partial}{\partial v}\left\{\frac{2^{v / 2}}{\Gamma(-v)}\right\} \sum_{k=0}^{A} T_{k}+\frac{2^{v / 2}}{\Gamma(-v)} \sum_{k=0}^{A} \frac{\partial T_{k}}{\partial v}
$$

The first sum $\sum T_{k}$ is given by Lemmas 4 and 5 . Consider the second sum

$$
\sum_{k=0}^{A} \frac{\partial T_{k}}{\partial v}=\sum_{k=0}^{M-1} \frac{\partial T_{k}}{\partial v}+\sum_{k=M}^{A} \frac{\partial T_{k}}{\partial v} \triangleq R_{\text {head }}+R_{\text {tail }}
$$

Here,

$$
\frac{\partial T_{k}}{\partial v}=[\ln \sqrt{a}-\psi(k-v)] T_{k}
$$

and $\psi(z)=\Gamma^{\prime}(z) / \Gamma(z)$. By [19, §6.3.5 and §6.3.2], [4, Eqs. 5.4.12, 5.4.14, and 5.5.2],

$$
\psi(k-v)=\psi(k)+O\left(\frac{1}{k}\right)=\ln k+O\left(\frac{1}{k}\right)=\ln k \cdot\left[1+O\left(\frac{1}{k \ln k}\right)\right]
$$

Define

$$
g_{v}(t) \triangleq \ln t \cdot f_{v}(t)=\ln t \cdot t^{-v-1} \exp \left(-\frac{t^{2}}{2}\right)=-\frac{\partial}{\partial v} f_{v}(t)
$$


Since $t \ln t \rightarrow 0$ when $t \rightarrow 0^{+}$, taking zero as the value at $t=0$, the functions $g_{v}(t)$ and

$$
g_{v}^{\prime \prime}(t)=\ln t \cdot f_{v}^{\prime \prime}(t)-\left(3+2 v+2 t^{2}\right) f_{v-2}(t)
$$

are continuous and bounded for bounded $v \leq-4$ and $t \geq 0$.

Lemma 8 For bounded $v \leq-4, R_{\text {tail }}=O(1 / \sqrt{a})$.

Proof By Lemmas 1 and 2, and (37),

$$
\begin{aligned}
0<R_{\text {tail }} & =\frac{\mathrm{d}}{\mathrm{d} v}\left\{a^{v / 2} \sum_{k=M}^{A} q(k) p(k)\right\} \\
& \leq a^{v / 2} \sum_{k=M}^{A}(\ln \sqrt{a}-\ln k) \cdot k^{-v-1}\left[1+O\left(\frac{1}{k}\right)\right] e^{-k^{2} / 2 A}\left[1+O\left(\frac{k}{a}\right)\right] \\
& =a^{v / 2} \sum_{k=M}^{A}(\ln \sqrt{a}-\ln k) \cdot k^{-v-1} e^{-k^{2} / 2 A} O(1) .
\end{aligned}
$$

But substituting $k=k \Delta t \sqrt{A}$,

$$
\begin{aligned}
R_{\mathrm{tail}} & =a^{v / 2} \sum_{k=M}^{A}[\ln \sqrt{a}-\ln (k \Delta t \sqrt{A})] \cdot(k \Delta t \sqrt{A})^{-v-1} e^{-(k \Delta t)^{2} / 2} \Delta t \sqrt{A} \cdot O(1) \\
& =\left(\frac{a}{A}\right)^{\frac{v}{2}} \sum_{k=M}^{A}\left[\ln \sqrt{\frac{a}{A}}-\ln (k \Delta t)\right] f_{v}(k \Delta t) \Delta t \cdot O(1) \\
& =-\sum_{k=M}^{A} g_{v}(k \Delta t) \Delta t \cdot O(1) .
\end{aligned}
$$

Since $|\ln t| \leq 1 / t$ for $0<t \leq 1$ and $|\ln t| \leq t$ for $t \geq 1,\left|g_{v}(t)\right| \leq f_{v+1}(t)+f_{v-1}(t)$ for $t \geq 0$, and for $v \leq-4$,

$$
\left|R_{\text {tail }}\right| \leq \sum_{k=M}^{A}\left[f_{v+1}(k \Delta t)+f_{v-1}(k \Delta t)\right] \Delta t \cdot O(1)=O\left(\frac{1}{\sqrt{a}}\right)
$$

by Lemma 3.

Lemma 9 For bounded $v \leq-4$,

$$
R_{\text {head }}=\frac{\mathrm{d}}{\mathrm{d} v}\left\{2^{-v / 2-1} \Gamma\left(-\frac{v}{2}\right)\right\}+O\left(\frac{1}{\sqrt{a}}\right)
$$


Proof This time $k<M$, and by Lemmas 1 and 2, like (20),

$$
\begin{aligned}
R_{\text {head }} & =\left(\frac{a}{A}\right)^{v / 2} \sum_{k=0}^{M-1}[\ln \sqrt{a}-\ln (k \Delta t \sqrt{A})](k \Delta t) \Delta t\left[1+O\left(\frac{1}{k}+\frac{k}{a}+\frac{k^{3}}{a^{2}}\right)\right] \\
& =-\sum_{k=0}^{M-1} g_{v}(k \Delta t) \Delta t+\sum_{k=0}^{M-1} g_{v}(k \Delta t) \Delta t \cdot O\left(\frac{\Delta t}{k \Delta t}+\frac{k \Delta t}{\sqrt{a}}+\frac{(k \Delta t)^{3}}{\sqrt{a}}\right) \\
& \triangleq S+\Delta S .
\end{aligned}
$$

Since $\left|g_{v}(t) t^{n}\right|=\left|g_{\nu-n}(t)\right| \leq f_{\nu-n+1}+f_{\nu-n-1}$, the error term $\Delta S$ is

$$
\begin{aligned}
|\Delta S| \leq & \sum_{k=0}^{M-1}\left[f_{v+2}(k \Delta t)+f_{v}(k \Delta t)\right] \Delta t \cdot O(\Delta t) \\
& +\sum_{k=0}^{M-1}\left[f_{v}(k \Delta t)+f_{v-2}(k \Delta t)\right] \Delta t \cdot O(1 / \sqrt{a}) \\
& +\sum_{k=0}^{M-1}\left[f_{v-2}(k \Delta t)+f_{v-4}(k \Delta t)\right] \Delta t \cdot O(1 / \sqrt{a})=O(1 / \sqrt{a})
\end{aligned}
$$

for $v \leq-4$ by Lemma 3 .

For the sum $S$ in (38), again using the trapezoidal rule, as in the proof of Lemma 3,

$$
\begin{aligned}
R_{\text {head }} & =-\sum_{k=0}^{M-1} g_{v}(t)(k \Delta t) \Delta t \\
& =-\int_{0}^{M \Delta t} g_{v}(t) \mathrm{d} t+O\left(\frac{1}{\sqrt{a}}\right) \\
& =\int_{0}^{\infty} \frac{\mathrm{d}}{\mathrm{d} v} f_{v}(t) \mathrm{d} t+\int_{M \Delta t}^{\infty} g_{v}(t) \mathrm{d} t+O\left(\frac{1}{\sqrt{a}}\right) .
\end{aligned}
$$

For $v \leq-4$

$\int_{M \Delta t}^{\infty}\left|g_{\nu}(t)\right| \mathrm{d} t \leq \int_{M \Delta t}^{\infty} f_{\nu+1}(t) \mathrm{d} t=2^{-(v+3) / 2} \Gamma\left(-\frac{v+1}{2}, \frac{(M \Delta t)^{2}}{2}\right)=O\left(\frac{1}{\sqrt{a}}\right)$

by (18) and (19).

The function $f_{v}$ satisfies $\int_{0}^{\infty} f_{v} \mathrm{~d} t<\infty$ for $v \leq-1$, and for $|h| \leq 1$ and $t \geq 1$,

$$
\left(\frac{t^{h}-1}{h}\right)=\left(\frac{e^{h \ln t}-1}{h}\right)=\ln t+\frac{\ln ^{2} t}{2 !} h+\frac{\ln ^{3} t}{3 !} h^{2}+\cdots \leq e^{\ln t}-1<t .
$$


The function $f_{v-1}(t)$ is an integrable function dominating $\left|f_{v+h}(t)-f_{v}(t)\right| / h$ for $|h| \leq 1$ and $t \geq 1$, since

$$
\left|\frac{f_{v+h}(t)-f_{v}(t)}{h}\right|=\left(\frac{t^{h}-1}{h}\right) f_{v}(t)<t f_{v}(t)=f_{v-1}(t),
$$

so by Lebesgue's dominant convergence theorem, the integration and differentiation order can be switched in the integral

$$
\int_{0}^{\infty} \frac{\mathrm{d}}{\mathrm{d} v} f_{v}(t) \mathrm{d} t=\frac{\mathrm{d}}{\mathrm{d} v}\left\{\int_{0}^{\infty} f_{v}(t) \mathrm{d} t\right\}=\frac{\mathrm{d}}{\mathrm{d} v}\left\{2^{-v / 2-1} \Gamma\left(-\frac{v}{2}\right)\right\} .
$$

Using Lemmas 9 and 8, Eq. (35) becomes

$$
\begin{aligned}
\frac{\mathrm{d}}{\mathrm{d} v} y_{v}^{a}(0)= & \frac{\mathrm{d}}{\mathrm{d} v}\left\{\frac{2^{v / 2}}{\Gamma(-v)}\right\} \cdot \sum_{k=0}^{A} T_{k}+\frac{2^{v / 2}}{\Gamma(-v)}\left(R_{\text {head }}+R_{\text {tail }}\right) \\
= & \frac{\mathrm{d}}{\mathrm{d} v}\left\{\frac{2^{v / 2}}{\Gamma(-v)}\right\} \cdot\left\{2^{-v / 2-1} \Gamma\left(-\frac{v}{2}\right)+O\left(\frac{1}{\sqrt{a}}\right)\right\} \\
& +\frac{2^{v / 2}}{\Gamma(-v)} \cdot\left\{\frac{\mathrm{d}}{\mathrm{d} v}\left[2^{-v / 2-1} \Gamma\left(-\frac{v}{2}\right)\right]+O\left(\frac{1}{\sqrt{a}}\right)\right\} \\
= & \frac{\mathrm{d}}{\mathrm{d} v}\left\{\frac{\Gamma\left(-\frac{v}{2}\right)}{2 \Gamma(-v)}\right\}+O\left(\frac{1}{\sqrt{a}}\right) \\
= & \frac{\mathrm{d}}{\mathrm{d} v} H_{v}(0)+O\left(\frac{1}{\sqrt{a}}\right) .
\end{aligned}
$$

\subsection{Convergence for $x=0$ and Arbitrary $v$}

Lemma 10 For $v$ in any bounded interval,

$$
\frac{\partial y_{v}(0)}{\partial v}=\frac{\partial H_{v}(0)}{\partial v}+O\left(\frac{1}{\sqrt{a}}\right)
$$

and for $\Delta x=1 / \sqrt{2 a}$,

$$
\frac{\partial}{\partial v}\left\{\frac{y_{v}(0)-y_{v}(-\Delta x)}{\Delta x}\right\}=\frac{\partial H_{v}^{\prime}(0)}{\partial v}+O\left(\frac{1}{\sqrt{a}}\right) .
$$

Proof Induction can be applied again, just as in the proof of Lemma 6. Given that $\partial y_{v}(0) / \partial v=\partial H_{v}(0) / \partial v+O(1 / \sqrt{a})$ for bounded $v<v_{0}$ and $n=A=\lceil a\rceil$, again using (22),

$$
\frac{\partial}{\partial v} y_{v+1}(0)=\frac{\partial}{\partial v}\left\{(2 a)^{(v+1) / 2} c_{A}(v+1)\right\}
$$




$$
\begin{aligned}
& =\frac{\partial}{\partial v}\left\{\sqrt{2 a} \frac{v+a-A}{a} y_{v}(0)-\frac{v}{a} 2 a y_{v-1}(0)\right\} \\
& =\frac{\sqrt{2}}{\sqrt{a}} \frac{\partial}{\partial v}\left\{v y_{v}(0)\right\}+\frac{(a-A) \sqrt{2}}{\sqrt{a}} \frac{\partial}{\partial v} y_{v}(0)+\frac{\partial}{\partial v}\left\{-2 v y_{v-1}(0)\right\} \\
& =O\left(\frac{1}{\sqrt{a}}\right)+\frac{\partial}{\partial v}\left\{-2 v y_{v-1}(0)\right\} \\
& =-2 y_{v-1}(0)-2 v \frac{\partial}{\partial v}\left\{y_{v-1}(0)\right\}+O\left(\frac{1}{\sqrt{a}}\right) .
\end{aligned}
$$

Applying the induction step,

$$
\begin{aligned}
\frac{\partial}{\partial v} y_{v+1}(0) & =-2 H_{v-1}(0)-2 v \frac{\partial}{\partial v}\left\{H_{v-1}(0)\right\}+O\left(\frac{1}{\sqrt{a}}\right) \\
& =\frac{\partial}{\partial v}\left\{-2 v H_{v-1}(0)\right\}+O\left(\frac{1}{\sqrt{a}}\right) \\
& =\frac{\partial}{\partial v}\left\{H_{v+1}(0)\right\}+O\left(\frac{1}{\sqrt{a}}\right) .
\end{aligned}
$$

This implies that $y_{v}(0)=H_{v}(0)+O(1 / \sqrt{a})$ locally for $v$. Using the backward recurrence relation (6), as in (24),

$$
\begin{aligned}
\frac{\partial}{\partial v}\left\{\frac{y_{v}(0)-y_{v}(-\Delta x)}{\Delta x}\right\} & =\frac{\partial}{\partial v} 2 v y_{v-1}(0) \\
& =2 y_{v-1}(0)+2 v \frac{\partial}{\partial v} y_{v-1}(0) \\
& =2 H_{v-1}(0)+2 v \frac{\partial}{\partial v} H_{v-1}(0)+O\left(\frac{1}{\sqrt{a}}\right) \\
& =\frac{\partial}{\partial v}\left\{2 v H_{v-1}(0)\right\}+O\left(\frac{1}{\sqrt{a}}\right) \\
& =\frac{\partial}{\partial v} H_{v}^{\prime}(0)+O\left(\frac{1}{\sqrt{a}}\right) .
\end{aligned}
$$

\subsection{Convergence for Arbitrary $x$ and Arbitrary $v$}

By differentiating Eq. (7) with respect to $v$, and defining $w \triangleq \partial y / \partial v$,

$$
w^{\prime \prime}=2 x w^{\prime}-2 v w-2 y=2 x w^{\prime}-2 v w-2 H_{v}(x) .
$$


This equation has the particular solution $w(x)=\partial H_{v}(x) / \partial v$. The homogeneous equation is again the Hermite equation, so the general solution of (39) is

$$
w=\frac{\partial H_{v}(x)}{\partial v}+A H_{v}(x)+B H_{v}(-x) .
$$

For initial conditions $w(0)=\partial H_{v}(0) / \partial v$ and $w^{\prime}(0)=\partial H_{v}^{\prime}(0) / \partial v$, the unique solution of (39) is obviously $w(x)=\partial H_{v}(x) / \partial v$.

Let $\boldsymbol{y}(x) \triangleq\left(y(x), y^{\prime}(x), w(x), w^{\prime}(x)\right)^{T}$. Equation (39) can be rewritten in the normal form (25), where

$$
\boldsymbol{A}(x) \triangleq\left(\begin{array}{llll}
0 & 1 & 0 & 0 \\
-2 v & 2 x & 0 & 0 \\
0 & 0 & 0 & 1 \\
-2 & 0 & -2 v & 2 x
\end{array}\right)
$$

This time, the Euclidean norm of $\boldsymbol{A}$ satisfies $\|\boldsymbol{A}(x)\| \leq \sqrt{6+8 v^{2}+8 x^{2}}$, giving the Lipschitz constant $\sqrt{6+8 \psi^{2}+8 \xi^{2}}$. In analogy with (26), a Cauchy polygon $\boldsymbol{u}(x)$ can be defined such that for $x$ and $v$ in bounded intervals $[0, \xi]$ and $[-\psi, \psi]$, respectively,

$$
\left|\frac{\partial(\boldsymbol{A}(x) \boldsymbol{u}(x))}{\partial x}\right|_{x=x_{k}}=\left|\left(\begin{array}{llll}
0 & 0 & 0 & 0 \\
0 & 2 & 0 & 0 \\
0 & 0 & 0 & 0 \\
0 & 0 & 0 & 2
\end{array}\right) \boldsymbol{u}_{k}\right| \leq 2\left|\boldsymbol{u}_{k}\right| \leq 2\left|\boldsymbol{u}_{0}\right| e^{L \xi}
$$

As in the proof of Lemma 7, this means that for bounded $x$ and $v, \boldsymbol{u}(x)$ converges uniformly to the solution $\boldsymbol{y}$ with an error bound

$$
|\boldsymbol{u}(x)-\boldsymbol{y}(x)| \leq O\left(\frac{1}{\sqrt{a}}\right) .
$$

Now, extending the definition of $\boldsymbol{z}_{k}(29)$ to four components, $\boldsymbol{z}_{0} \triangleq \boldsymbol{u}_{0}$ and

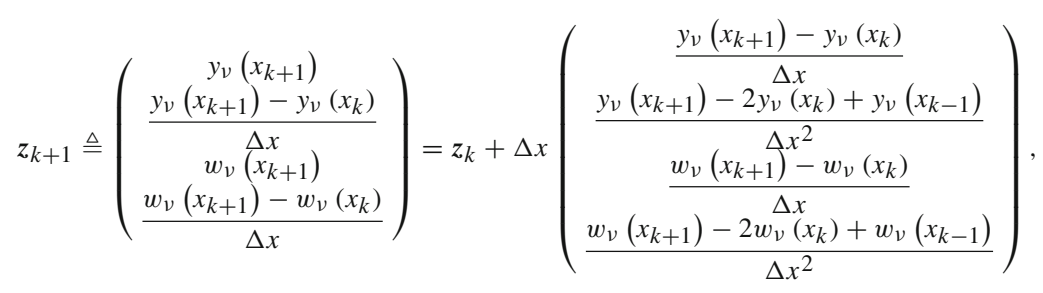

and writing $d_{m} \triangleq \partial c_{m} / \partial v$,

$$
z_{k}=\left(\begin{array}{c}
r^{v} c_{m} \\
r^{v+1}\left(c_{m-1}-c_{m}\right) \\
r^{v} d_{m} \\
r^{\nu+1}\left(d_{m-1}-d_{m}\right)
\end{array}\right)
$$


and

$$
z_{k+1}=z_{k}+\Delta x\left(\begin{array}{c}
r^{\nu+1}\left(c_{m-1}-c_{m}\right) \\
r^{\nu+2}\left(c_{m-1}-2 c_{m}+c_{m+1}\right) \\
r^{\nu+1}\left(d_{m-1}-d_{m}\right) \\
r^{\nu+2}\left(d_{m-1}-2 d_{m}+d_{m+1}\right)
\end{array}\right)
$$

Differentiating (30) with respect to $v$,

$r^{2} d_{m+1}-2 r^{2} d_{m}+r^{2} d_{m-1}=2 x_{k} r\left(d_{m-1}-d_{m}\right)-2 v d_{m}-2 \theta\left(d_{m-1}-d_{m}\right)-2 c_{m}$,

leads to

$$
\begin{aligned}
z_{k+1} & =z_{k}+\Delta x\left(\begin{array}{cccc}
0 & 1 & 0 & 0 \\
-2 v & 2 x_{k}-2 \theta / r & 0 & 0 \\
0 & 0 & 0 & 1 \\
-2 & 0 & -2 v & 2 x_{k}-2 \theta / r
\end{array}\right) z_{k} \\
& =z_{k}+\Delta x \boldsymbol{A}\left(x_{k}\right) z_{k}+\Delta x\left(\begin{array}{cccc}
0 & 0 & 0 & 0 \\
0 & -2 \theta / r & 0 & 0 \\
0 & 0 & 0 & 0 \\
0 & 0 & 0 & -2 \theta / r
\end{array}\right) z_{k} .
\end{aligned}
$$

By a procedure similar to the application of Eqs. (31)-(33) in Sect. 2,

$$
\left|\frac{\partial y_{v}^{a}(x)}{\partial v}-\frac{\partial H_{v}(x)}{\partial v}\right| \leq O\left(\frac{1}{\sqrt{a}}\right)
$$

where the right hand side is independent of $x$ and $v$ for these parameters in any bounded interval.

The sharpness of the bound can be proved by contradiction: Suppose that $\partial y_{v}^{a}(x) / \partial v-\partial H_{v}(x) / \partial v=O(b(a))$ where $O(b(a))$ is tighter than $O(1 / \sqrt{a})$. Integrating this difference,

$\int_{\nu_{1}}^{v_{2}}\left[\frac{\partial y_{v}^{a}(x)}{\partial v}-\frac{\partial H_{v}(x)}{\partial v}\right] \mathrm{d} v=\left[y_{\nu_{2}}^{a}(x)-H_{\nu_{2}}(x)\right]-\left[y_{\nu_{1}}^{a}(x)-H_{\nu_{2}}(x)\right]=O(b(a))$.

Choosing $v_{1}=0$ and $v_{2}=2$, arbitrary $x$, arbitrarily large $a$ such that $a-x \sqrt{2 a}$ is integer, and using (34),

$$
\left[y_{2}^{a}(x)-H_{2}(x)\right]-\left[y_{0}^{a}(x)-H_{0}(x)\right]=\frac{2 x \sqrt{2}}{\sqrt{a}}-(1-1)=\frac{2 x \sqrt{2}}{\sqrt{a}},
$$

which is a contradiction. This completes the proof of Theorem 4. 


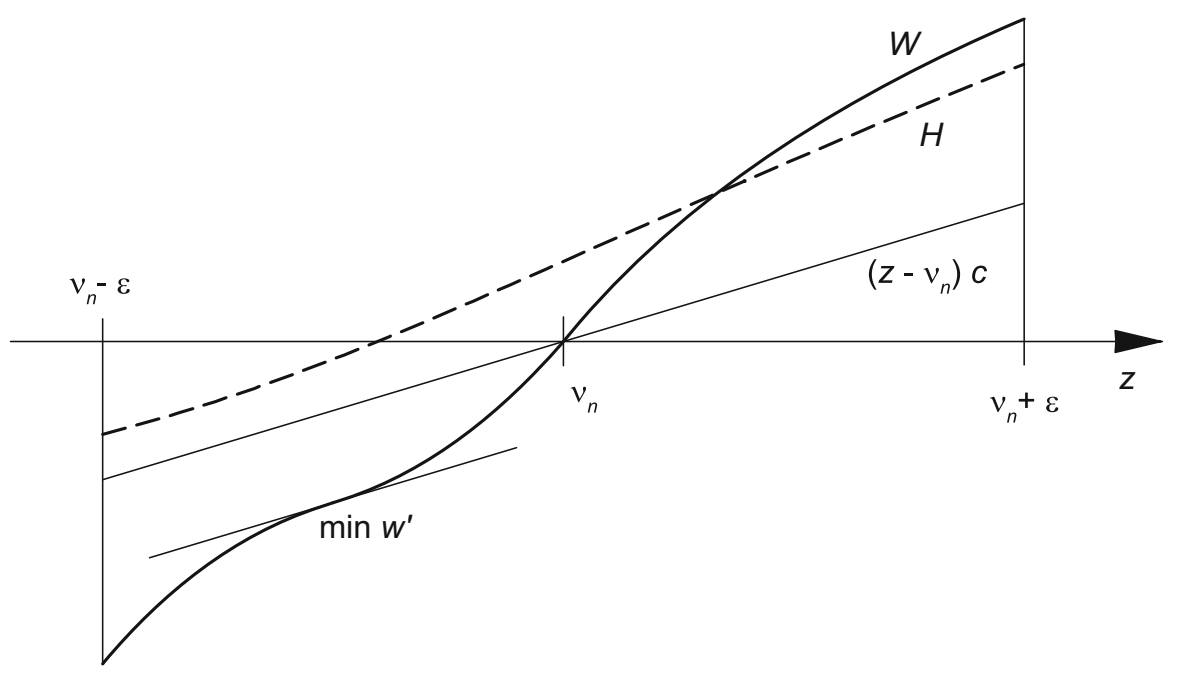

Fig. 3 The Hermite function must have a zero near the Charlier polynomial zero

\section{Convergence of Zeros}

Theorem 5 For fixed real $x$ and positive $a \rightarrow \infty$, let $n \triangleq\lceil a-x \sqrt{2 a}\rceil$. For $a$ convergent sequence of zeros $v_{n} \rightarrow v$ such that $c_{n}^{a}\left(v_{n}\right)=0$, the limit $v$ is a zero of the Hermite function, $H_{v}(x)=0$, satisfying $v=v_{n}+O(1 / \sqrt{a})$. Conversely, for a positive real zero $v$ of the Hermite function, there is a convergent sequence $v_{n} \rightarrow v$ of zeros of $c_{n}^{a}$ satisfying $v=v_{n}+O(1 / \sqrt{a})$.

Proof Define $w_{n}(z) \triangleq(\sqrt{2 a})^{n} c_{n}^{a}(z)$ and note that $w_{n}$ has the same zeros in $z$ as $c_{n}^{a}$. The proof is based on the well-known fact that the zeros of a Charlier polynomial are real, simple, and positive [8]. Taylor-expanding $w_{n}(z)$ around one of its zeros $z=v_{n}$, writing $w_{n}^{\prime}\left(v_{n}\right)$ for $\partial w_{n}(z) / \partial z$ at $z=v_{n}$, $w_{n}\left(v_{n}+\varepsilon\right)=w_{n}\left(v_{n}\right)+\varepsilon w_{n}^{\prime}\left(v_{n}\right)+O\left(\varepsilon^{2}\right)=\varepsilon\left(w_{n}^{\prime}\left(v_{n}\right)+O(\varepsilon)\right) \triangleq \varepsilon W\left(v_{n}, \varepsilon\right)$.

Since the zeros of a Charlier polynomial are simple, $w_{n}^{\prime}\left(v_{n}\right) \neq 0$, the expression $W\left(v_{n}, \varepsilon\right)$ must be nonzero for $\varepsilon$ in some sufficiently small interval $I=[-\delta, \delta]$, where $0<\delta \leq v_{n}$. Assume that $w_{n}^{\prime}\left(v_{n}\right)>0$. The case $w_{n}^{\prime}\left(v_{n}\right)<0$ is treated in an analog way. Let $c \triangleq \inf _{\varepsilon \in I} W\left(v_{n}, \varepsilon\right)$. Figure 3 illustrates $\left|\left(z-v_{n}\right) c\right|$ as a lower bound for $\left|w_{n}(z)\right|$. By Theorem 1, due to the uniform convergence, for $z \in\left[v_{n}-\delta, v_{n}+\delta\right]$, there is a $b$, independent of $n$ and $z$, such that

$$
\left|H_{z}(x)-w_{n}(z)\right| \leq \frac{b}{\sqrt{a}}
$$


Choose $\varepsilon \triangleq(1+b) /(c \sqrt{a})$, which satisfies $\varepsilon<\delta$ for sufficiently large $a$. For $z=v_{n}+\varepsilon$,

$$
H_{z}(x) \geq w_{n}(z)-\frac{b}{\sqrt{a}}=\varepsilon W\left(v_{n}, \varepsilon\right)-\frac{b}{\sqrt{a}} \geq \frac{1+b}{c \sqrt{a}} c-\frac{b}{\sqrt{a}}=\frac{1}{\sqrt{a}}>0 .
$$

Similarly, $z=v_{n}-\varepsilon$ implies that $H_{z}(x)<0$. Since $H_{z}(x)$ is an entire function and changes sign for $\mathrm{z}$ in $\left[v_{n}-\varepsilon, v_{n}+\varepsilon\right]$, it must have a zero there. By letting $a \rightarrow \infty$, the theorem is proved in one direction. For the reverse direction, switch the roles of $w$ and $H$. Assume that $H_{v}(x)=0$. Since $H_{0}(x) \equiv 1, v$ cannot be zero. Expand $H_{z}(x)$ around $z=v$, writing $\partial H_{v}(x) / \partial v$ for $\partial H_{z}(x) / \partial z$ at $z=v$,

$$
H_{v+\varepsilon}(x)=H_{v}(x)+\varepsilon \partial H_{v}(x) / \partial v+O\left(\varepsilon^{2}\right)=\varepsilon\left(\partial H_{v}(x) / \partial v+O(\varepsilon)\right) \triangleq \varepsilon Z(v, \varepsilon) \text {. }
$$

Let $x(v)$ be defined as the $p$ th zero in $x$ of $H_{v}(x)=0$. It is known that $x(v)$ is a strictly monotonic function of $v$ for $v \geq 0$, so $\mathrm{d} x / \mathrm{d} v \neq 0$ [21]. Differentiating the equation by $v$,

$$
\frac{\partial H_{v}(x)}{\partial v}+\frac{\partial H_{v}(x)}{\partial x} \frac{\mathrm{d} x}{\mathrm{~d} v}=0
$$

so obviously, $\partial H_{v}(x) / \partial v=0$ if and only if $\partial H_{v}(x) / \partial x=0$. But if the latter derivative is zero, then $H_{v-1}(x)=0$ by the derivative rule (9), and according to the three-term recurrence for Hermite functions (8), all derivatives of $H_{z}(x)$ would be zero at $z=v$, entailing that $H$, being analytic, would be identically zero. In other words, all positive real zeros $v$ of $H_{v}(x)$ are simple.

Consequently, $\partial H_{v}(x) / \partial v \neq 0$, and similarly to the first half of the proof, $Z(v, \varepsilon)$ must be nonzero for $\varepsilon$ in some sufficiently small interval. It follows that $w_{n}(z)$ must be zero for some $z \in[v-\varepsilon, v+\varepsilon]$, where $\varepsilon=O(1 / \sqrt{a})$.

\section{Conclusions}

It has been shown that the scaled Charlier polynomials, their scaled derivatives, and zeros converge to the Hermite function, the derivative of the Hermite function, and the zeros of the Hermite function, respectively. The convergence rates are inversely proportional to the square root of the order of the Charlier polynomial.

The proof technique used for showing the convergence of Charlier polynomials and their first derivatives is applicable to higher derivatives of the polynomials. There is a possibility that the Charlier polynomials may be extensible to an entire function. Such an extension could simplify the convergence proofs, but finding it appears nontrivial.

Acknowledgements This research was funded by the European Union FP7 research project THE, "The Hand Embodied," under Grant Agreement 248587. The author is grateful for support by Prof. Henrik Jörntell of Lund University, Department of Experimental Medical Science.

Funding Open access funding provided by RISE Research Institutes of Sweden. 
Open Access This article is licensed under a Creative Commons Attribution 4.0 International License, which permits use, sharing, adaptation, distribution and reproduction in any medium or format, as long as you give appropriate credit to the original author(s) and the source, provide a link to the Creative Commons licence, and indicate if changes were made. The images or other third party material in this article are included in the article's Creative Commons licence, unless indicated otherwise in a credit line to the material. If material is not included in the article's Creative Commons licence and your intended use is not permitted by statutory regulation or exceeds the permitted use, you will need to obtain permission directly from the copyright holder. To view a copy of this licence, visit http://creativecommons.org/licenses/by/4.0/.

\section{References}

1. Charlier, C.V.L.: Über die Darstellung willkürlicher Funktionen. Ark. Mat. Astron. Fys. 2(20), 1-35 (1905)

2. Erdélyi, A., Magnus, W., Oberhettinger, F., Tricomi, F.G.: Higher Transcendental Functions, vol. II. McGraw-Hill, New York, Toronto, London (1953)

3. Szegő, G.: Orthogonal Polynomials, AMS Colloquium Publications, vol. XXIII, 4th edn. American Mathematical Society, Providence (1975)

4. Olver, F.W.J., Lozier, D.W., Boisvert, R.F., Clark, C.W. (eds.): NIST Handbook of Mathematical Functions. Cambridge University Press, New York (2010)

5. Koekoek, R., Lesky, P.A., Swarttouw, R.F.: Hypergeometric Orthogonal Polynomials and Their $q$ Analogues. Springer, Berlin (2010)

6. Jagerman, D.L.: Some properties of the Erlang loss function. Bell Syst. Tech. J. 53(3), 525-551 (1974)

7. Karlin, S., McGregor, J.: Many server queueing processes with Poisson input and exponential service times. Pac. J. Math. 8(1), 87-118 (1958)

8. Kijima, M.: On the largest negative eigenvalue of the infinitesimal generator associated with $M / M / n / n$ queues. Oper. Res. Lett. 9, 59-64 (1990)

9. Nilsson, M.N.P.: Hitting time in Erlang loss systems with moving boundaries. Queueing Syst. 78(3), 225-254 (2014). https://doi.org/10.1007/s11134-014-9399-5

10. Engel, D.D.: The Multiple Stochastic Integral, Memoirs of the American Mathematical Society, vol. 38, pp. 1-82. AMS, Providence (1982)

11. Xiu, D.: Numerical Methods for Stochastic Computations: A Spectral Method Approach. Princeton University Press, Princeton (2010)

12. König, W.: Orthogonal polynomial ensembles in probability theory. Probab. Surv. 2, 385-447 (2005). arXiv:math/0403090v3 [math.PR]

13. Halfin, S., Whitt, W.: Heavy-traffic limits for queues with many exponential servers. Oper. Res. 29(3), 567-588 (1981)

14. Meixner, J.: Erzeugende Funktionen der Charlierschen Polynome. Math. Z. 44(1), 531-535 (1939). https://doi.org/10.1007/BF01210670

15. Lebedev, N.N.: Special Functions and Their Applications. Dover Publications, New York (1972)

16. Dominici, D.: Asymptotic analysis of the Askey scheme I: from Krawchouk to Charlier. Cent. Eur. J. Math. 5(2), 280-304 (2007)

17. Dunster, T.M.: Uniform asymptotic expansions for Charlier polynomials. J. Approx. Theory 112, 93-133 (2001). https://doi.org/10.1006/jath.2001.3595

18. Laforgia, A.: Further inequalities for the gamma function. Math. Comput. 42(166), 597-600 (1984)

19. Abramowitz, M., Stegun, I.A.: Handbook of Mathematical Functions. Applied Mathematics Series 55. National Bureau of Standards, Washington, DC (1972)

20. Birkhoff, G., Rota, G.-C.: Ordinary Differential Equations, 2nd edn. Xerox College Publishing, Lexington (1969)

21. Elbert, A., Muldoon, M.E.: Inequalities and monotonicity properties for zeros of Hermite functions. Proc. R. Soc. Edinb. Sect. A 129, 57-75 (1999)

Publisher's Note Springer Nature remains neutral with regard to jurisdictional claims in published maps and institutional affiliations. 